\title{
Ökonomisierung aus wirtschaftswissenschaftlicher Perspektive
}

\author{
Jürgen Heinrich
}

Ökonomisierung wird aus wirtschaftswissenschaftlicher Perspektive als Zunabme monetärer und egoistischer Elemente in der Nutzenfunktion der Wirtschaftssubjekte interpretiert. Die Ursache der Ökonomisierung ist der Wandel des Leitbildes der Wirtschaftspolitik und der Medienpolitik sowie der zunehmende Wettbewerb. Die Ökonomisierung ist auf der Ebene des Individuums, der Unternebmung, des Marktes und der Politik beobachtbar. Wesentliche Folge ist die Zunabme der allokativen und der produktiven Effizienz der Medienindustrie.

Ökonomisierung wird von denjenigen beklagt, die schwindendes Einkommen und schwindenden Einfluss fürchten, und sie wird von denjenigen gefordert, die sich davon eine Verbesserung ihrer Situation versprechen. Im Folgenden wird das Konzept der Ökonomisierung im Abschnitt 1 beschrieben, in Abschnitt 2 werden die Ursachen der Ökonomisierung herausgestellt und in Abschnitt 3 werden die Ebenen und Instrumente der Ökonomisierung der Medienindustrie analysiert. Eine sehr kurze Spekulation über weiter gehende Folgen der Ökonomisierung bietet abschließend der Abschnitt 4.

\section{Zum Konzept der Ökonomisierung}

Vorab sei betont, dass Ökonomisierung als ein Prozess verstanden werden sollte, als ein Prozess der Zunahme der Bedeutung des „Ökonomischen“ im Verhalten der Individuen; grundlegend ökonomisch geprägt ist das Verhalten der Menschen spätestens seit der Einführung der Tausch- und der Geldwirtschaft schon immer gewesen. Grundlegende Denkmuster der Ökonomie sind

- die Annahme eines rationalen Handelns der Individuen, konkretisiert in der „ökonomischen Ethik“ der Kosten-Nutzen-Analyse (Boulding 1973, S. 117) und

- die Akzeptanz des Wettbewerbs als grundsätzlich optimales Verfahren zur Maximierung der individuellen Wohlfahrt.

Beide Denkmuster sollten das Konzept der Ökonomisierung fundieren, obwohl sie logisch unterschiedlichen Ebenen zuzuordnen sind: Als Ökonomisierung bezeichne ich die Zunahme monetärer und egoistischer Elemente in der Nutzenfunktion der Wirtschaftssubjekte und eine zunehmend striktere Anwendung des Nutzenmaximierungspostulats. Beides wird durch die Zunahme des Wettbewerbs induziert. Einen Unterschied zum Konzept der Kommerzialisierung zu machen, wie es bisweilen gehandhabt wird, erscheint mir nicht sinnvoll. Kommerz/Kommerzialisierung scheint mir die bisweilen abwertend gemeinte Bezeichnung für Ökonomisierung zu sein, meist im Gegensatz zu Kultur (vgl. z. B. Kunczik 1992 oder Schmitz/Tompert 1995). Eine solche Wertung ist möglich, trägt indes nicht zu einer Klärung des Konzeptes bei.

Differenziert man nach den Ebenen der Ökonomisierung, so wird das Konzept deutlicher und operationaler. Die Ökonomisierung manifestiert sich vor allem auf der Ebene des Individuums, der Unternehmung, des Marktes und der Politik. Auf der Ebene des Individuums gewinnen im Prozess der Ökonomisierung monetäre und egoistische Elemente, wie z. B. monetäres Einkommen und persönliche Annehmlichkeit, an Gewicht gegenüber nichtmonetären und altruistischen Elementen, wie z. B. die Befriedi- 
gung durch die Erfüllung intrinsischer Motivationen zum gedachten Wohle der Allgemeinheit (zur schwierigen Unterscheidung zwischen altruistischem und egoistischem Verhalten vgl. Kirchgässner 1996, S. 226 ff.). Auf der Ebene der Unternehmung manifestiert sich die Ökonomisierung in dem Bestreben, die Gewinne durch eine immer billigere und/oder besser auf die Präferenzen der Konsumenten abgestellte Produktion zu steigern. Auf der Ebene des Marktes manifestiert sich die Ökonomisierung vor allem in einer Zunahme des Wettbewerbs und auf der Ebene der Politik in einer zunehmenden Akzeptanz des Wettbewerbs als Problemlösungsverfahren (vgl. auch Meckel 1999, S. $129 \mathrm{ff}$.$) .$

Als Merkmale der Ökonomisierung der Medienindustrie können mithin folgende Verschiebungen angesehen werden, die stets als graduelle Veränderungen interpretiert werden sollten:

- Ein Ersatz der publizistischen Ziele von Aufklärung, Kritik und Kontrolle durch marktorientierte Ziele,

- eine zunehmende Berücksichtigung von Rezipientenpräferenzen („Umdefinition des Bürgers zum Konsumenten“, Hoffmann-Riem, 1988, S. 59),

- ein Abbau der Quersubventionierung reichweitenschwacher Medienangebote,

- eine Annäherung an Kostenpreise (insbesondere durch Pay-Rundfunk),

- eine Annäherung an Gewinnmaßstäbe und ein Ersatz der nichtgewinnorientierten Erfolgsmaßstäbe durch den Shareholder-Value und

- eine Annäherung an die Zurechnung von Handlungsfolgen vor allem durch die Privatisierung öffentlicher Institutionen und durch zunehmend exakter werdende Reichweitenmessungen.

Demgegenüber haben weniger ökonomische Verfahren, wie z. B. die Gebührenfinanzierung des Rundfunks, eine mehr oder weniger begrenzte Autonomie, die Kosten der Produktion und/oder die Präferenzen der Rezipienten zu missachten. Sie können andere Ziele verfolgen, wie z. B. das Gemeinwohl, oder die Ziele umfassend verstandener Stakeholder, und/oder sie unterliegen als öffentliche Unternehmen nicht den strengen Regeln des Insolvenzrechts, müssen also nicht alle Handlungsfolgen tragen.

\section{Ursachen der Ökonomisierung}

Ursache der Ökonomisierung ist vor allem der Wandel des Leitbilds der Wirtschaftspolitik: Die Abkehr vom konstruktivistischen Rationalismus, der darauf gesetzt hatte, dass eine rationale politische Steuerung von Marktprozessen notwendig und möglich sei, und die Hinwendung zum offenen Wettbewerb als Entdeckungsverfahren. Dies Leitbild des offenen Wettbewerbs, der klassischen Wettbewerbsfreiheit, setzt vor allem und je nach Ausprägung auch ausschließlich auf die Freiheit, Wettbewerb zu veranstalten, konkret also auf die Freiheit des Marktzutritts. Wichtig ist es in dieser Sicht, Marktzutrittsschranken abzubauen; die resultierende Marktstruktur ist dann von allenfalls geringer Bedeutung. Konzentration von Marktmacht ist per se bedeutungslos, sie wird kritisch nur dann beurteilt, wenn sie eine substanzielle Marktzutrittsschranke darstellt. Exakt in diesen Kontext ist die neu in das deutsche Kartellrecht aufgenommene „Essential Facilities Doctrine“ einzuordnen. Als Behinderungsmissbrauch gilt die Weigerung, „einem anderen Unternehmen gegen angemessenes Entgelt Zugang zu den eigenen Netzen oder anderen Infrastruktureinrichtungen zu gewähren, wenn es dem anderen Unternehmen aus rechtlichen oder tatsächlichen Gründen ohne die Mitbenutzung nicht möglich ist, auf dem vor- oder nachgelagerten Markt als Wettbewerber des marktbeherrschenden Unternehmens tätig zu werden“ (\$19, Abs. 4, Punkt 4 KartellG). Diese Dok- 
trin ist sehr wirkungsvoll, um den Marktzutritt von Konkurrenten zu erleichtern, eine bestehende Verflechtung wird eher ignoriert.

Warum sich das Leitbild der Wirtschaftspolitik gewandelt hat, ist nicht leicht zu klären. Es ist zu vermuten, dass der geringe Charme der Marktalternativen, also ihre hohen Kosten und ihre geringe Effizienz, den Wandel des Leitbilds der Wirtschaftspolitik induziert hat.

Ursache der Ökonomisierung ist zum anderen die spezifische Ausprägung des technischen Fortschritts: die Abnahme der Distanzüberwindungskosten relativ zu den Produktionskosten und die Digitalisierung der Informationen. Die Abnahme der Distanzüberwindungskosten führt zu einer Ausdehnung der Märkte bis hin zu einer Globalisierung von Märkten. Diese Ausdehnung der Märkte verstärkt den Wettbewerb, sie wirkt wie ein Marktzutritt neuer Wettbewerber. Vor wenigen Jahren war die Deutsche Bundespost der einzige Anbieter auf dem Markt für Telekommunikation, jetzt konkurrieren mindestens fünf Anbieter und weitere Marktzutritte sind unschwer möglich. Die Digitalisierung verstärkt den Wettbewerb noch einmal, weil - etwa durch B 2 B und B 2 C - die Markttransparenz erhöht, die Märkte vergrößert und die Macht der Nachfrager gestärkt werden.

Für die Medienindustrie ist die Globalisierung indes weniger relevant als für die industrielle Produktion insgesamt. Medienmärkte bleiben räumlich stark begrenzte Märkte und alle Globalisierungsversuche müssen die Präferenzen für räumlich segmentierte Informationen überwinden. Das Handelsblatt und offenbar auch die Financial Times müssen in Deutschland produziert werden, um in Deutschland gelesen zu werden, und dabei ist die Wirtschaft noch der Bereich, der am leichtesten globalisierbar wäre. Die Digitalisierung allerdings revolutioniert gerade die Medienindustrie:

- Es ist ihr Rohstoff, mit dem neue Wertschöpfungsprozesse gefüllt werden und der zur Branchenkonvergenz führt;

- es ist ein neuer Bereich der Wirtschaft entstanden, der von den alten Regulierungsstrukturen gar nicht tangiert werden konnte und kann und in dem neue Wettbewerbskräfte freigesetzt worden sind.

Dennoch kann als Ursache der Ökonomisierung der Medienindustrie vor allem der Wandel des Leitbilds der Wirtschaftspolitik und nachfolgend der Medienpolitik vermutet werden. Einer rationalen Medienpolitik muss bewusst werden, dass eine Abweichung vom Markt bzw. vom Wettbewerbsmodell deswegen nicht durchzuhalten ist, weil weiteste Teile der Medienproduktion sich nicht durch ihre Produktion, sondern erst durch ihre Rezeption legitimieren und weil es Normadressaten für die Rezeption gesellschaftlich erwünschter Medienproduktion nicht gibt - allenfalls in der Schule oder im Gefängnis. Es reicht eben nicht, das Angebot des meritorischen Gutes „Integrationsrundfunk" und anderer gesellschaftlich erwünschter Medienangebote zu subventionieren - so wie es vielleicht bei Schulmilch gehandhabt werden kann - um die Rezeption zu fördern, aber andere Mittel zur Beeinflussung stehen nicht zur Verfügung.

\section{Ebenen der Ökonomisierung}

Die Ökonomisierung manifestiert sich auf der Ebene des Individuums, der Unternehmung, des Marktes und der Politik. Diese Trennung erfolgt vor allem aus analytischen Gründen, in der faktischen Produktion der Medienindustrien verwischen sich diese Ebenen. Die Veränderungen in diesen Ebenen werden im Folgenden beschrieben, dabei liegt der Schwerpunkt auf der Ebene der Unternehmung. 
Auf der Ebene des Individuums manifestiert sich die Ökonomisierung in einer immer strikteren Anwendung der persönlichen Kosten-Nutzen-Analyse und einem entsprechenden Rückgang von Handlungsweisen, die einer von Boulding so genannten „heroischen Ethik“ folgen. Boulding unterscheidet drei Hauptformen der heroischen Ethik: Die militärische Ethik „Frag nicht warum, tu deine Pflicht und stirb“, die religiöse Ethik „Geben und nicht nach den Kosten fragen, arbeiten und nicht nach der Belohnung verlangen" als Gebet des Heiligen Franziskus und die sportliche Ethik, die im Grunde mit der ökonomischen Ethik der Kosten-Nutzen-Analyse nicht in Einklang zu bringen sind (Boulding 1973, S. 118 ff.). Und auch die alte journalistische Ethik, die etwa durch die gewissenhafte Erfüllung der öffentlichen Aufgabe des Journalismus (vgl. Branahl 1981) beschrieben werden kann, entzieht sich der ökonomischen Ethik der strikten KostenNutzen-Analyse und wird durch andere Berufsnormen ersetzt, die eher einem egoistischen Nutzenmaximierungskalkül entsprechen. Über diesen Wandel der Berufsauffassung liegen Studien vor, die genau diese Form individueller Ökonomisierung bei Journalisten belegen (z. B. Bridges 1991, Weischenberg 1995, S. 330 ff. oder Blöbaum 2000). Dabei kann in neoklassischer ökonomischer Denktradition (vgl. insbesondere Becker 1993) diese beobachtbare Verhaltensänderung von Journalisten sehr gut - bei Konstanz der Präferenzstrukturen von Journalisten - mit einer Veränderung von Kosten bzw. Preisen der Erfüllung der öffentlichen Aufgabe gegenüber den Kosten bzw. Preisen eines mehr an Unterhaltung und privatem Nutzwert orientierten Journalismus erklärt werden: Die Kosten eines Aufklärungsjournalismus steigen relativ, während seine Bezahlung relativ abnimmt, und für den Unterhaltungs-/Nutzwertjournalismus ist es umgekehrt.

Auf der Ebene der Unternehmung manifestiert sich die Ökonomisierung am deutlichsten. Hier wird die Ökonomisierung geplant, organisiert und umgesetzt. Movens ist die immer striktere Anwendung der Kosten-Nutzen-Analyse in der unternehmensspezifischen Form des Ziels der Gewinnmaximierung oder, wie heute präzisiert wird, in Form der Maximierung des Shareholder-Values, des Gewinns für die Anteilseigner (vgl. Matthes 2000). Erzwungen durch die Anlagestrategien der großen Vermögensfonds und durch die zunehmende Kontrolle der Unternehmung durch Finanz-Analysten spielen andere Ziele keine Rolle mehr, oder, genauer gesagt, werden dem Shareholder-Value untergeordnet.

Auch die Ansprüche der heute oft genannten Stakeholder, denen früher eine eigenständige Rolle zuerkannt worden ist, wurden mit der zunehmenden Ökonomisierung dem Shareholder-Value untergeordnet. Als Stakeholder einer Unternehmung bezeichnet man jene Gruppen der Gesellschaft, die die Unternehmung beeinflussen und/oder von ihr beeinflusst werden, also z. B. Kunden, Lieferanten Arbeitnehmer, Kreditgeber oder der Staat als Subventionszahler und Steuerempfänger. Und es ist durchaus wichtig für eine Unternehmung, die Beziehungen zu diesen Gruppen befriedigend zu gestalten, wenn die Unternehmung langfristig auf dem Markt bestehen will (vgl. Zentes 1998, S. 333). Aber diese Ansprüche werden zunehmend nur soweit berücksichtigt, wie sie der Maximierung des Shareholder-Values zuträglich sind.

Im Rahmen der Gewinnmaximierung bzw. der Maximierung des Shareholder-Values lassen sich zwei Bereiche recht gut unterscheiden, die im Prozess der Ökonomisierung der Unternehmung die entscheidende Rolle spielen:

Zum einen wachsen die Anstrengungen der Unternehmen, die so genannte allokative Effizienz zu steigern, also durch Produktinnovationen die Produktqualität immer mehr den Konsumentenpräferenzen anzupassen und/oder durch Werbung die Konsumentenpräferenzen zu beeinflussen. Dieser Komplex kann dem betrieblichen Funktions- 
segment des Marketings zugeordnet werden und kann auch als Qualitätswettbewerb bezeichnet werden (Heinrich 1996, S. 165).

Zum anderen wachsen die Anstrengungen der Unternehmen, die so genannte produktive Effizienz zu steigern, also durch Prozessinnovationen einschließlich betrieblicher Reorganisationen eine effizientere Produktionsweise zu erreichen. Dieser Komplex kann dem betrieblichen Funktionssegment des Managements zugeordnet werden und kann auch als Kostenwettbewerb bezeichnet werden (Heinrich 1996, S. 166).

Wird die allokative Effizienz gesteigert, wird stets genauer das produziert, was die Rezipienten verlangen. Die Rezipienten verlangen einen Informationsnutzen und/oder einen Animationsnutzen, einen Unterhaltungswert. Es resultiert dann ein Unterhaltungs- und ein Gebrauchswertjournalismus zu Lasten von Aufklärung und Investigation. Weischenberg spricht hier von Marketingjounalismus (Weischenberg 1995, S. 334 ff.). „RTL 2“ und „Focus“ mögen als Beispiele genügen.

Die werbungtreibende Wirtschaft, der zweite und wichtigere Kunde der Medienunternehmen, verlangt ebenfalls einen Nutzen, eine Verbreitungs- und Wirkungswahrscheinlichkeit von Werbebotschaften: nämlich eine Reichweite, einen Zielgruppenbezug und ein wirksames Werbeumfeld. Dies ist nicht nur negativ zu werten. Billiger Sex, Ekel-TV und sehr blutige Gewalt eignen sich nicht als Werbeumfeld, und die Glaubwürdigkeit des Mediums wird sowohl von der werbungtreibenden Wirtschaft als auch von den Rezipienten geschätzt. Prinzipiell ist die Orientierung der Medienproduktion auf die Werbung indes doch problematisch, weil Umfang und Zielgruppen der Berichterstattung werblich definiert werden und nicht nach den Präferenzen des Publikums. Es resultiert ein Werbeumfeldjournalismus, sowohl in bestehenden Medien als auch in der Konzeption neuer Medienangebote, insbesondere bei Zeitschriften und bei Spartenprogrammen.

In einem solchen Ökonomisierungsprozess, der auf eine genauere Berücksichtigung von Rezipientenpräferenzen setzt, schwinden naturgemäß reichweitenschwache Programme und auch Inhalte, die Rezipientenpräferenzen missachten. Als Beispiel sei die Entwicklung der „Welt“ aus dem Axel Springer Verlag gewählt, auch, um deutlich zu machen, dass eine Ökonomisierung nicht per se negativ gewertet werden kann. Die „Welt“ war (auch) Verbreitungsmedium der konservativen Grundhaltung des Verlegers Axel Springer, der die Produktion der „Welt“ erheblich mit den Erlösen aus rezipientenfreundlicheren Blättern subventionierte. Mittlerweile ist die „Welt" liberaler und auch erfolgreicher, also ökonomischer geworden, und die zunehmende Liberalität wird gemeinhin positiv beurteilt.

Wird die produktive Effizienz gesteigert, so wird versucht, billiger zu produzieren. Insbesondere folgende Maßnahmen sind geeignet, Kosten der Medienproduktion zu senken:

- Die Einführung von Kontrollsystemen, etwa die Einführung einer Prozesskostenrechnung, einer Deckungsbeitragsrechnung oder von Profitcentern, erhöht die Wirtschaftlichkeit der Medienproduktion. Jede Maßnahme wird daraufhin zu überprüfen sein, ob ihr Grenzumsatz ihre Grenzkosten übersteigt, ob also als Differenz ein Grenzgewinn, ein zusätzlicher Gewinn erzielt werden kann. Ein solcher Grenzgewinnjournalismus hat eine Tendenz zum Billigjournalismus, weil die Grenzkosten im Regelfall recht genau kalkuliert werden können; der Grenzumsatz ex ante hingegen nicht.

- Die Ausgliederung der Produktion in den Markt, das so genannte Outsourcing, spart Kosten, weil der Markt im Prinzip billiger produziert als die eigene Unternehmung. Dies führt zu einem Kaufjournalismus, dessen Qualität kaum noch kontrolliert werden kann und der die publizistische Vielfalt verringert. 
- Der Aufbau von Verwertungsketten im Rahmen eines gut kalkulierten Content Managements erlaubt die Kosten sparende bzw. einnahmesteigernde Mehrfachverwertung von Informationsinhalten, die sich in der Rezeption ja nicht verbrauchen. Man spricht von der Nichtrivalität im Konsum und in der Medienindustrie von Mehrfachverwertung, und nachfolgend von Kaskadenjournalismus, eine stete Verbreiterung und Verflachung gleicher Inhalte (vgl. Heinrich 1999, S. 45).

Die Ökonomisierung auf Unternehmensebene kann ansatzweise empirisch erfasst werden. Das Endergebnis der Steigerung von allokativer und produktiver Effizienz sollte eine Steigerung der Profitrate von Medienunternehmen sein. Einige deutsche Fernsehveranstalter verzeichnen mittlerweile jedenfalls beachtliche und steigende Umsatzrenditen. RTL erzielte 1999 eine Umsatzrendite vor Steuern von 16,7\% nach 7,9\%, 9,3\%, 10,3\% und 13,7\% in den Jahren 1995 bis 1998. Oder die ProSieben-Gruppe erzielte 1999 eine Umsatzrendite vor Steuern von 16\% nach $8 \%, 11 \%, 14 \%$ und $16 \%$ in den gleichen Vorjahren (Veranstalterangaben von RTL und ProSieben). Allerdings muss beachtet werden, und methodisch wäre dies schwer zu separieren, dass die Zunahme des Wettbewerbs, die effizienzsteigernde Maßnahmen erzwingt, langfristig zu einer Nivellierung der Profitrate führen sollte.

Auf den Vorstufen könnte möglicherweise der Input des Marketing- und Managementaufwands und ihr jeweiliger Output mit Hilfe geeigneter Indikatoren erfasst werden. So könnte der Input des Marketingaufwands von Medienunternehmen durch den jeweiligen Anteil der Beschäftigten im Längsschnitt und im Querschnitt ermittelt werden. Nach den Erhebungen des DIW liegt z. B. der Beschäftigungsanteil für das Marketing im privaten Rundfunk bei 11 bis 17 Prozent der Beschäftigten und zwar mit steigender Tendenz (DLM 1997, S. 70 ff.; DLM 2000, S. 59), wohingegen dieser Anteil beim öffentlich-rechtlichen Rundfunk praktisch bei Null Prozent liegt (DLM 2000, S. 83 f.). Oder der Output zunehmender Marketinganstrengungen könnte durch die Veränderung der Zahl der Titel von Zeitschriften, die Zunahme der Zahl der Programme, der Formate und auch der Medien selbst, etwa der Online-Medien, erfasst werden. Um nur eine Zahl zu nennen: Allein im März 1998 starteten 38 neue Programmformate im Fernsehen, z. B. „Brigitte TV“ in der ARD oder „Warm up“ bei DSF (Media Facts 3/1998, S. 13). Auch die Gestalt und die Inhalte von Medienproduktionen ändern sich und dies könnte ansatzweise durch Programmanalysen erfasst werden (vgl. z. B. Krüger 2000 und Weiß 1999).

Der Markt ist die Ebene, die die Ökonomisierung vorantreibt und erzwingt, weil sich im Wettbewerb diejenigen Unternehmen durchsetzen, die die Ökonomisierung am schnellsten und umfassendsten umsetzen - bei "Strafe des Untergangs“ (Marx). Im Markt manifestiert sich die Ökonomisierung vor allem in folgenden Bereichen: In der Zunahme der Konzentration, die in der rasch expandierenden Medienindustrie statistisch noch nicht recht zu erfassen ist, die aber in der Fülle von Unternehmensverbindungen sichtbar wird (jüngstes Beispiel ist der Zusammenschluss von ProSieben und SAT.1 und das letztlich resultierende Kirch-Bertelsmann-Duopol). Zahl und Volumen der M \& A-Transaktionen in der Medienindustrie scheinen kontinuierlich anzusteigen, so ist die Zahl der M \& A-Transaktionen in Europa von 1999 auf 2000 um 164,5 Prozent gestiegen und das Volumen um 3,5 Prozent (Andersen 2000). In diesen Konzentrationsprozessen werden zum einen Synergieeffekte der Produktion und zum anderen Synergieeffekte der Vermarktung angestrebt und zum Teil auch realisiert. Konzernjournalismus ist die Folge.

Deutlich sichtbar wird im Markt daneben eine Zunahme der Vermarktungsebenen bzw. der Wertschöpfungsstufen und -ebenen. Zu nennen sind zurzeit die Ausweitung 
der Werbung in die nicht-klassische Werbung „below the line“, also Sponsoring, Bartering, Product Placement, Infomercials, Storymercials, Splitscreen, virtuelle Werbung usw., sowie eine Zunahme des Merchandising, eine Zunahme der Mehrfachverwertung und der Versuch, Informationen nach ihrem Wert gestaffelt zu verkaufen.

Auf der Ebene der Politik zeigt sich die Ökonomisierung ganz allgemein in einer sehr umfangreichen Deregulierung vor allem der Telekommunikation und der Versorgungsunternehmen. Diese Deregulierung erstreckt sich auch auf die Medienindustrie, einerseits, insofern sie Teil des Telekommunikationssektors ist, und andererseits insofern, als die Regelungen des Medienrechts durch die Regelungen des Wirtschaftsrechts insbesondere des Kartellrechts langsam ersetzt werden bzw. ersetzt werden sollen (vgl. z. B. Kommunikationsordnung 2000 und Gutachten des wissenschaftlichen Beirats beim BMWi).

\section{Folgen der Ökonomisierung}

Folgen der Ökonomisierung sind zunächst einmal die Folgen zunehmenden Wettbewerbs. Diese sind mit der Zunahme von allokativer und produktiver Effizienz, mit der Zunahme von Konzentration und Wertschöpfungsebenen und mit dem Wandel der Medienpolitik zur Wirtschaftspolitik beschrieben worden. Auf der Ebene journalistischen Handelns ergibt sich ein Trend zu einem Unterhaltungs-, Nutzwert-, Werbeumfeld-, Grenzgewinn-, Kauf-, Konzern- und Kaskadenjournalismus, wobei fraglich ist, ob so gekennzeichnete Medienproduktionen generell noch als journalistische Produktionen bezeichnet werden sollten. Über weiter gehende Folgen der Ökonomisierung zu spekulieren, bleibt anderen Disziplinen vorbehalten. $\mathrm{Zu}$ vermuten ist, dass damit auch dem Konzept und der Funktionsweise von Öffentlichkeit eine andere Bedeutung zukommt (vgl. Imhoff/Jarren/Blum 2000) oder dass die Massenmedien ihre soziale Bildungs- und Integrationsfunktion verändert erfüllen.

\section{Literatur}

Andersen, Arthur (2000): Deal Survey 2000.

Becker, Gary S. (1993): Ökonomische Erklärung menschlichen Verhaltens, 2. Aufl., Tübingen.

Blöbaum, Bernd (2000): Strukturwandel des Journalismus - Strukturwandel von Öffentlichkeit, in: K. Imhoff, O. Jarren, R. Blum (Hrsg.): Zerfall der Öffentlichkeit, Mediensymposium Luzern Bd. 6, Opladen.

Boulding, Kenneth. E. (1973): Ökonomie als eine Moralwissenschaft, in: W. Vogt (Hrsg.): Seminar Politische Ökonomie, Frankfurt, S. 103 - 125.

Branahl, Udo (1981): Öffentliche Aufgabe, in: K. Koszyk, K. H. Pruys (Hrsg.): Handbuch der Massenkommunikation, München, S. $211-214$.

Bridges, Janet A. (1991): Daily newspaper managing editors perception of news media functions, in: Jounalism Quarterly, Vol. 68, S. 718 - 728.

DLM (1997): Beschäftigte und wirtschaftliche Lage des Rundfunks in Deutschland 1995/96: Studie des Deutschen Instituts für Wirtschaftsforschung (DIW) in Kooperation mit dem Hans-Bredow-Institut und der Arbeitsgruppe Kommunikationsforschung München (AKM), Berlin.

DLM (2000): Beschäftigte und wirtschaftliche Lage des Rundfunks in Deutschland 1997/98: Studie des Deutschen Instituts für Wirtschaftsforschung (DIW) in Kooperation mit dem HansBredow-Institut und der Arbeitsgruppe Kommunikationsforschung München (AKM), Berlin.

Heinrich, Jürgen (1996): Qualitätswettbewerb und/oder Kostenwettbewerb im Mediensektor, in: Rundfunk und Fernsehen, 44. Jg., S. 165 - 184.

Heinrich, Jürgen (1999): Medienökonomie Bd. 2: Hörfunk und Fernsehen, Opladen. 
Hoffmann-Riem, Wolfgang (1988): Rundfunkrecht und Wirtschaftsrecht - ein Paradigmawechsel in der Rundfunkverfassung?, in: Media Perspektiven, 2, S. 57 - 72.

Imhoff, Kurt; Jarren, Ottfried; Blum, Roger (Hrsg.) (2000): Zerfall der Öffentlichkeit, Mediensymposium Luzern Bd. 6, Opladen.

Kirchgässner, Gebhard (1996): Bemerkungen zur Minimalmoral, in: Zeitschrift für Wirtschaftsund Sozialwissenschaften, 116, S. $223-251$.

Kommunikationsordnung 2000 (1997): Grundsatzpapier der Bertelsmann Stiftung zu Leitlinien zukünftiger Kommunikationsordnung, Gütersloh.

Krüger, Udo Michael (2000): Unterschiedliches Informationsverständnis im öffentlich-rechtlichen und privaten Fernsehen, in: Media Perspektiven 7/2000, S. 278 - 296.

Kunczik, Michael (1992): Massenmedien und Gesellschaft. Theoretische Modelle unter besonderer Berücksichtigung des Aspekts Kultur und Kommerz, in: Privat-kommerzieller Rundfunk in Deutschland, Bonn (Bundeszentrale für politische Bildung), S. 13 - 30.

Matthes, Jürgen (2000), Das deutsche Corporate-Governance-System, Köln.

Meckel, Miriam (1999): Redaktionsmanagement, Opladen.

Media Facts 3/1998

Schmitz, Hermann-Josef; Hella Tompert (1995): Kommerz kontra Kultur? Hohenheimer Protokolle Bd. 49, Stuttgart.

Weischenberg, Siegfried (1995): Journalistik 2, Medientechnik, Medienfunktionen, Medienakteure, Opladen.

Weiß, Hans-Jürgen (1999): Programmalltag in Deutschland, in: ALM (Hrsg.): Programmbericht zur Lage und Entwicklung des Fernsehens in Deutschland, Berlin, S. 69-126.

Wissenschaftlicher Beirat beim Bundesministerium für Wirtschaft und Technologie (1999): Offene Medienordnung, Berlin.

Zentes, Joachim (1998): Marketing, in: Vahlens Kompendium der Betriebswirtschaftslehre, Bd. 1, 4. Aufl., München, S. $329-409$. 